\title{
Capacity Rationing of Product in Advance Selling
}

\author{
Yan Zhao ${ }^{1, a^{*}}$ Taofeng Ye $\mathrm{Y}^{1, \mathrm{~b}}$ \\ ${ }^{1}$ School of Economics and Management, Jiangsu University of Science and Technology, Zhenjiang, Jiangsu 212000, \\ China \\ a*18261958070@163.com \\ bytf_jj@163.com
}

\begin{abstract}
We consider a retailer who sells a single product to strategic consumers sensitive to price over two periods: advance and spot. The retailer's decisions include whether to offer the product and, if so, the prices in both periods, and whether to ration capacity in the advance period. This article takes the retailer's profit maximization as the goal, and focusing on how the retailer determines the capacity rationing strategy when selling products to strategic consumers, and backward induction is used to derive the seller's decisions in equilibrium. Research indicates that the retailer's optimal rationing policy is unrelated with the cost of product, and is mainly determined by the retailer's capacity and the number of consumers who arrive in advance.
\end{abstract}

Keywords: capacity rationing, advance selling, strategic consumer behavior

\section{预售情境下产品的能力配给}

\author{
赵妍 $^{1, a^{*}}$ 叶涛锋 ${ }^{1, b}$
}

${ }^{1}$ 江苏科技大学经济管理学院, 镇江, 江苏, 中国

a*18261958070@163.com

${ }^{b} y t f \_j j @ 163 . c o m$

\section{摘要}

我们考虑一个产能有限的零售商, 在两个阶段（预售和现货）向对价格敏感的策略性消费者销售单一 产品。零售商的决策包括是否提供产品, 如果提供, 则应如何确定最优的两个阶段的价格, 以及是否 提前定量分配产能。本文以零售商利润最大化为目标, 研究零售商向策略消费者销售产品时, 如何确 定能力配给策略, 利用逆向归纳来推导卖方在均衡状态下的决策。研究结果表明, 零售商的预售决策 与成本无关，主要由零售商的产能和提前到达的消费者数量共同决定的。

关键词: 能力配给; 预售; 策略性消费者行为

\section{1. 引言}

预售是指零售商在消费之前就向顾客提供购买承 诺的机会的一种营销实践（Xie and Shugan 2009） [1]。 近年来, 这种经营策略在多种行业中普遍存在, 如娱乐 业 (音乐会和体育赛事) 、服务业 (酒店和航空) 和电 子产品 (手机和电脑) 等。随着信息技术的发展, 消费 者获取产品信息的方式越来越多, 这使得消费者更具有 策略性。在预售环境中, 策略型消费者通过比较预售期 和现货期的消费者剩余来决定其购买行为, 因此, 零售 商在制定经营策略时, 必须考虑消费者估值不确定和可 获得性对策略型消费者行为的影响。而且 Chevalier 和

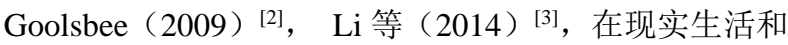
实验室中都广泛发现了策略型消费者行为。由于策略型 消费者行为非常普遍, 所以将策略型消费者视为近视型 可能会造成零售商的利润损失，如 Nair（2007） ${ }^{[4]}$ 称， 忽视策略型消费者行为而损失的利润可能大于 $20 \%$ 。

在当前的研究中, 我们考虑一个零售商在两个时期 内向策略型消费者提供单一产品：预售期和现货期，现 货期也是消费期。与有关预售的文献相比，我们假设零 售商在能力配给方面具有灵活性, 在这种情况下, 零售 商的决策变量包括预售期和现货期的价格, 在零售商在 预售期提供的产品数量。鉴于在预售期开始时预售价格 以及零售商提供的数量, 提前到达的消费者可以通过预 测现货期的价格和可获得性来决定是否以及何时购买 产品。 
本研究的主要研究问题如下。零售商是否预售? 如 果预售, 则零售商在预售期是否满足全部需求? 在预售 期和现货期如何定价?

\section{2. 模型}

\section{1 零售商决策}

零售商需要决定是否提供预售, 如果提供, 则预售 价格为 $p_{1}$, 预售数量为 $S$ 。此外, 无论是否提供预售, 零售商都需要决定现货价格 $p_{2}$ 。

\section{2 消费者决策}

市场规模为确定性的消费者数量组成, 其中 $N_{1}$ 个 消费者在预售期到达, $N_{2}\left(N_{2}=N-N_{1}\right)$ 个消费者在现 售期到达。在此, $N_{1}$ 是指在预售期知道产品发布的消 费者数量, 而 $N_{2}$ 个消费者仅在现售期（或预售期即将 结束时) 得知产品发布。换句话说, $N_{1}$ 个消费者可能 对该产品更感兴趣, 并对它的研发和上市更关注。尽管 预售期的潜在需求可以确定, 但是预售期和现货期的实 际需求及其分配取决于零售商在这两个时期内的价格, 预售期产能分配的决定。同样，在现货期到达并决定购 买产品的消费者数量由零售商对现货价格的决策决定。

假定每个消费者都是相互独立的, 因此可以忽略它 们之间的相互影响。 $N_{1}$ 个消费者到达后, 他们会根据 预售期的价格和可获得性以及他们对现货期的价格和 最终可获得性的估计来决定是否以及何时购买产品。我 们假设消费者拥有完整的信息, 因此会对公司的决策形 成合理的期望, 也就是说, 消费者的预估与均衡结果是 一致的。对于那些在现货期到达的消费者, 他们只需要 决定是否购买, 因为他们没有其他机会获得产品。由于 到零售商的产能 $T$ 和预售期到达的消费者数量 $N_{1}$, 零 售商的预售政策为以下三种类型之一： $S=0$ 代表不预 售, $S \in\left(0, \min \left\{T, N_{1}\right\}\right)$ 代表 部 分预售, 以及 $S=\min \left\{T, N_{1}\right\}$ 代表全部预售。

消费者决定是否购买和何时购买是基于他们在不 同时期获得产品的预期效用。我们假设顾客对价格很敏 感, 他们的边际支付意愿是异质的。在时间 $t(t=1,2$ 分别指预售期和现售期), 考虑到单价 $p_{t}, \theta$ 类型的消 费者消费一个单位的产品获得的效用为： $u\left(\theta, p_{t}\right)=\theta-p_{t}$ 。 $\theta$ 在 $[0,1]$ 上服从均匀分布。零售 商和提前到达的消费者虽然不确定这些消费者的类型, 但了解其分布, 消费者类型在现货期间显示。根据零售 商的决策, 消费者通过使其预期效用最大化来决定是否
以及何时购买产品。每个消费者最多购买一件产品。在 给定的零售商配给政策 $S$ 和销售价格 $p_{t}$ 的情况下, 消 费者在 $t$ 期购买产品实际得到的概率为 $\lambda_{t}\left(p_{t}, S\right)$ 。显 然, $\lambda_{t}\left(p_{t}, S\right)$ 可以表示为:

$$
\begin{aligned}
& \lambda_{1}\left(p_{1}, S\right)=\min \left\{1, \frac{S}{N_{1}\left(1-p_{1}\right)}\right\} \text { 和 } \\
& \lambda_{2}\left(p_{2}, S\right)=\min \left\{1, \frac{T-S}{(N-S)\left(1-p_{2}\right)}\right\} .
\end{aligned}
$$

估值为 $\theta$ 的消费者在时期 1 和 2 购买产品的期望效 用分别是:

$$
\begin{gathered}
u_{1}\left(p_{1}, S\right)=\lambda_{1}\left(p_{1}, S\right) E_{\theta}\left(\theta-p_{1}\right) \\
+\left[1-\lambda_{1}\left(p_{1}, S\right)\right] E_{\theta} \max \left[\lambda_{2}\left(p_{2}, S\right)\left(\theta-p_{2 q}, 0\right)\right], \\
u_{2}\left(p_{2}, S\right)=E_{\theta} \max \left[\lambda_{2}\left(p_{2}, S\right)\left(\theta-p_{2}, 0\right)\right] \\
\text { 由 于 } u_{2}\left(p_{2}, S\right) \geq 0, \text { 于 是 当 且 仅 当 } \\
u_{1}\left(p_{1}, S\right) \geq u_{2}\left(p_{2}, S\right) \text { 时消费者将提前购买, 得出 } \\
p_{1} \leq E_{\theta}\left\{\theta-\max \left[\lambda_{2}\left(p_{2}, S\right)\left(\theta-p_{2}, 0\right)\right]\right\}(2.1)
\end{gathered}
$$

\section{3. 最优决策求解}

在本节中, 我们讨论有限产能下零售商的最优决策, 包括定价, 是否提供预售以及预售期的能力配给。下面, 我们先讨论零售商在现货期的决策, 再讨论零售商在预 售期的决策

\section{1 现货期}

考虑到零售商在预售期的能力配给决策 $S$, 在现货 期想要购买的消费者数量为 $(N-S)\left(1-p_{2}\right)$ 。零售商 的产能是有限的, 因此一些潜在的需求可能会得不到满 足。给定总产能 $T$, 现货期初剩余产能为 $T-S$ 。因此, 现货期实际需求为 $\min \left\{T-S,(N-S)\left(1-p_{2}\right)\right\}$ 。 此时, 现货期的期望利润可表示为: $\prod_{2 q}^{C}\left(p_{2}, S\right)=\left(p_{2}-c\right) \min \left\{T-S,(N-S)\left(1-p_{2}\right)\right\}$, 零 售商的最优现货价格如下引理所示:

引理 3.1 由于 $\theta \in[0,1]$, 因此销售价格也需要满 足 $p \in[0,1]$, 并且只有当 $c \in[0,1]$ 零售商才会提供产 品。定义

$$
p_{2}^{B}(S)=\frac{N-T}{N-S}, \quad p_{2}^{U}=\frac{1+c}{2}
$$


零售商的最优现货价格可表示为:

$$
p_{2}^{C}=\left\{\begin{array}{cc}
p_{2}^{B}(S) & \text { 若 } T-S \leq(N-S)\left(1-p_{2}^{U}\right) \\
p_{2}^{U} & \text { 若 } T-S \geq(N-S)\left(1-p_{2}^{U}\right)
\end{array} .\right.
$$

从引理 3.1 的证明可知, $T-S \leq(N-S)\left(1-p_{2}^{U}\right)$ 等价于 $p_{2}^{B}(S)>p_{2}^{U}$ 。由式(8) 可知, 最优现货价格是 现货期间零售商剩余产能, $T-S$, 的递减函数。由于 假设残值为零, 当产能较大时, 零售商会降低现货价格 来清仓。

\section{2 预售期}

给定最优现货价格 $p_{2}^{C}$, 零售商的预售期目标是在 $p_{1}$ 和 $S$ 下, 使得利润最大化:

$\Pi^{C}\left(p_{1}, S\right)=\left(p_{1}-c\right) S+\left(p_{2}^{C}-c\right) \min \left\{T-S,(N-S)\left(1-p_{2}^{C}\right)\right\}$ 显然, $\Pi^{C}\left(p_{1}, S\right)$ 是 $p_{1}$ 的增函数。根据式 $(2.1)$, 最 优预售价格可以表示为:

$$
p_{1}^{C}=E_{\theta}\left\{\theta-\max \left[\lambda_{2}\left(p_{2}^{C}, S\right)\left(\theta-p_{2}^{C}, 0\right)\right]\right\} .
$$

为了得到最优的 $p_{1}$, 我们需要推导出现货期的需 求满足率。通过将 $p_{2}^{C}$ 带入到 $\lambda_{2}\left(p_{2}, S\right)$, 对于 $T-S \leq(N-S)\left(1-p_{2}^{U}\right)$ 和 $T-S \geq(N-S)\left(1-p_{2}^{U}\right)$ 我们都 有:

$$
\lambda_{2}\left(p_{2}^{C}, S\right)=1 \text {. }
$$

这表明，在现货期间的需求满足率与剩余产能无关。 换句话说, 零售商确定现货价格使得任何想要购买并且 能够支付现货价格的消费者都可以获得产品。

将式 $\lambda_{2}\left(p_{2}^{C}, S\right)=1$. 代入 $p_{1}^{C}$, 得到:

$$
\begin{aligned}
p_{1}^{C} & =E_{\theta} \min \left\{p_{2}^{C}, \theta\right\} \\
& =\left\{\begin{array}{cc}
p_{1}^{B}(S) & \text { 若 } T-S \leq(N-S)\left(1-p_{2}^{U}\right) \\
p_{1}^{U} & \text { 若 } T-S \geq(N-S)\left(1-p_{2}^{U}\right)
\end{array}\right.
\end{aligned}
$$

其 $\quad$ 中 $\quad p_{1}^{B}(S)=\frac{(N-T)(N+T-2 S)}{2(N-S)^{2}}$ $p_{1}^{U}=\frac{-c^{2}+2 c+3}{8}$

因此 $p_{1}^{C} \leq p_{2}^{C}$; 也就是说, 在产能有限的情况下, 零售商提供打折预售。

将式(3.1) 代入 $\Pi^{C}(S)$, 得关于 $S$ 的单变量利润
函数如下:

$\Pi^{C}(S)=\left(p_{1}^{C}-c\right) S+\left(p_{2}^{C}-c\right) \min \left\{T-S,(N-S)\left(1-p_{2}^{c}\right)\right\}$. 令 $T-S=(N-S)\left(1-p_{2}^{U}\right)$, 我们得到一个 $S$, 设 为 $\bar{S}=N-\frac{N-T}{p_{2}^{U}}$. 因此, $\Pi^{C}(S)$ 可以改写成 $\Pi^{C}(S)=\left\{\begin{array}{ll}\Pi^{B}(S) & \text { 若 } S \geq \bar{S} \\ \Pi^{U}(S) & \text { 若 } S \leq \bar{S}\end{array}\right.$, 其中: $\Pi^{B}(S)=\left\{E_{\theta} \min \left[p_{2}^{B}(S), \theta\right]-c\right\} S$ $+\left[p_{2}^{B}(S)-c\right](T-S)$,

$\Pi^{U}(S)=\left[E_{\theta} \min \left(p_{2}^{U}, \theta\right)-c\right] S+\left(p_{2}^{U}-c\right)(N-S)\left(1-p_{2}^{U}\right)$ $=\frac{\prod^{U}\left(N_{1}\right)-\prod^{U}(0)}{N_{1}} S+\left(p_{2}^{U}-c\right)\left(1-p_{2}^{U}\right) N$.

在 $S=\bar{S}$ 处, $\Pi^{C}(\bar{S})=\Pi^{B}(\bar{S})=\Pi^{U}(\bar{S})$ 。

为了求出零售商最优得配给策略, 我们需要先得出 $\Pi^{C}(S)$ 关于 $S$ 的变化趋势。

$$
\begin{aligned}
\Pi^{U}(S)= & {\left[\left(p_{1}^{U}-c\right)-\left(p_{2}^{U}-c\right)\left(1-p_{2}^{U}\right)\right] S } \\
& +N\left(p_{2}^{U}-c\right)\left(1-p_{2}^{U}\right)
\end{aligned}
$$

显然, $\prod_{q}^{U}\left(S_{q}\right)$ 是 $S_{q}$ 的单调函数。其单调性取决 于上式等号右边方括号项的值是正是负。由此可以推断, 零售商提供全预售或只在现货期出售产品是最优的, 即 $S_{q}^{U}=0$ 或 $S_{q}^{U}=N_{1}$ 。由 于 $\left(p_{1}^{U}-c\right)-\left(p_{2}^{U}-c\right)\left(1-p_{2}^{U}\right)=\left(-3 c^{2}-2 c+1\right) / 8$, 当 $c \leq \frac{1}{3}$ 时, $\left(p_{1}^{U}-c\right)-\left(p_{2}^{U}-c\right)\left(1-p_{2}^{U}\right) \geq 0$ ；当 $c>\frac{1}{3}$ 时, $\left(p_{1}^{U}-c\right)-\left(p_{2}^{U}-c\right)\left(1-p_{2}^{U}\right)<0 \quad$ ，所以 $\Pi^{C}(S)=\Pi^{U}(S)$ 时, 零售商最优策略的具体情况如 下命题 3.1 所示:

命题 3.1 零售商的最优策略如下: 当 $c \leq \frac{1}{3}$ 时, 提 供全预售; 当 $c>\frac{1}{3}$ 时, 不预售。

$\Pi^{B}(S)$ 的性质在下面的命题 3.2 中给出。

命题 3.2. 令 $\tilde{S}_{q}=\frac{2 N(2 T-N)}{N+T}, \widehat{S}_{q}=\bar{N}_{1}=\frac{N(3 T-N)}{N+T}$ 
当产能是中等时, 即 $\frac{N}{3}<T \leq \bar{T}$, 现货价格可能过高 导致所有产品不能在现货期间销售完。为了增加实际需 求和售空产品, 现在零售商将选择满足预售期的需求。 如果需求相对较低, 所有的预售需求都会得到满足。但 是, 当预售消费者数量较大时, 零售商会在预售期间对 产能进行定量配给, 以保留足够的产能在现货期间以较 高的价格出售。最后, 当产能足够大时, 即 $T>\bar{T}$, 零 售商必须提供全预售，即满足所有预售期需求，以出售 尽可能多的产品。

此外, $\Pi^{B}(S)$ 最大值在如下点处取得:

$$
S^{B}=\left\{\begin{array}{cc}
0, & \text { 若 } T \leq \frac{N}{3} \\
\min \left\{T, N_{1}\right\}=N_{1}, & \text { 若 } \frac{N}{3}<T \leq \bar{T}, N_{1} \leq \bar{N}_{1} \\
\widehat{S}<\min \left\{T, N_{1}\right\}, & \text { 若 } \frac{N}{3}<T \leq \bar{T}, N_{1}>\bar{N}_{1} \\
\min \left\{T, N_{1}\right\}=N_{1}, & \text { 若 } T>\bar{T}
\end{array} .\right.
$$

\section{项目基金}

本文为江苏省研究生科研创新计划项目《预售情境 下产品的质量选择与能力配给》(KYCX19_1649) 的阶段 性成果之一。

\section{REFERENCES}

命题 3.2 表明 $\Pi^{B}(S)$ 的图像性质和最优值取决于 $T$ 的值。因为当零售商在现货期的剩余产能不是很大时, 即 $\Pi^{C}(S)$ 等于 $\Pi^{B}(S)$ 时, 可以推断出零售商关于能 力配给的最优决策也依赖于 $T$. 结合命题 3.1 和命题 3.2 可得零售商的最优策略如下命题 3.3 所示。

命题 3.3 零售商在产品供应和能力配给方面的最优 决策可定义为:

- $T<\frac{N}{3}$. 零售商只在现货期提供产品;

- $\frac{N}{3}<T \leq \bar{T}$. 当 $N_{1} \leq \bar{N}_{1}$ 时, 零售商全预售; 当 $N_{1}>\bar{N}_{1}$. 零售商提供预售数量为 $\widehat{S}_{H}$ ；

- $T>\bar{T}$. 零售商提供全预售;

\section{4. 结论}

命题 3.3 表明零售商的预售决策与成本无关, 主要 由零售商的产能和提前到达的消费者数量共同决定的。 当总产能非常低以至于只有小部分到达的消费者能够 得到满足（即 $T \leq \frac{N}{3}$ ) 时, 因为零售商需要提供打折 预售，所以该产品只在现货期间提供，这样所有提前到 达的消费者都不得不推迟购买。为了以更高的价格出售 尽可能多的产品, 零售商会选择将所有的预售期的需求 推迟到现货期。该结论与预售期到达的消费者数量无关。

[1] Xie, J., S.M. Shugan. 2009. Advance selling theory. V.R. Rao eds. Handbook of Pricing Research in Marketing. Edward Elgar publishers.

[2] Chevalier, J., A. Goolsbee. 2009. Are durable goods consumers forward-looking? Evidence from college textbooks. Quarterly Journal of Economics 124(4) 1853-1884.

[3] Li, J., N. Granados, S. Netessine. 2014. Are consumers strategic? Structural estimation from the air-travel industry. Management Science 60(9) 2114-2137.

[4] Nair, H. 2007. Intertemporal price discrimination with forward-looking consumers: Application to the US market for console video-games. Quantitative Marketing and Economics 5 239-292. 